\title{
VIBRATION DOSE VALUES FOR WHOLE-BODY VIBRATION: SOME EXAMPLES
}

\author{
M.J. Griffin \\ Human Factors Research Unit \\ Institute of Sound and Vibration Research \\ The University \\ Southampton \\ SO9 $5 \mathrm{NH}$
}

SUMMARY

The vibration recorded in a car, vans, truck, tractor, tank, helicopters, fixed wing aircraft, hovercraft, hydrofoil, ship, and a building have been assessed according to both the current ISO 2631 procedures and a proposed revised method of indicating vibration severity. The revised method offers frequency weightings for assessing discomfort produced by multiaxis vibration. It also defines a vibration dose procedure which can be used to consider the probability of any adverse health effects of low or high crest factor continuous or intermittent vibration or repeated shock.

The revised procedure worked well in all 22 examples examined. It is shown that it may normally be used with root mean square measures of appropriately weighted vibration. However, for the more complex conditions where this is not appropriate an entirely compatible simple alternative calculation procedure is available. Some of the limitations of the current ISO procedure which necessitate its revision are discussed in relation to the proposed revised method.

\section{INTRODUCTION}

An understanding of the time-dependency of human responses to vibration is vital for the definition of procedures for assessing vibration severity. The magnitude of vibration is often expressed by either a peak or an average measure. Such values are particularly convenient when tests are conducted to compare the vibration generated in two or more controlled

Paper presented at the United Kingdom Informal Group Meeting, on Human Response to Vibration held at Heriot-Watt University, Edinburgh, 21/22 September 1984. 
conditions. The peak value reflects the conditions at only one instant of time and its use implies that any lower value occurring for any duration will have no effect on response. The use of a peak value would theirefore imply that human response is unaffected by the duration of the vibration. An average value may also be unaffected by changes in the measurement period: if the vibration is ergodic then, by definition, the time-average is the same for all portions of the signal. However, all time-averaging procedures contain an implicit time dependency whose effects becomes apparent when the conditions are non-ergodic. If the conditions can be assumed to be ergodic a time dependency can be defined by specifying different 'Iimits' for the average measure for different durations of exposure.

It is self-evident that many of the vibration conditions encountered in occupational and leisure activities are non-ergodic. Time averaging is then only valid if the implicit time-dependency is appropriate or the exposure is divided into a series of sections each having ergodic characteristics. Such sub-division may often prove both cumbersome and arbitary. Different results will be possible for the same exposure if the time-dependency applied to assess the importance of the duration of each section is different from the time-dependency used to average the values within the sections. Furthermore, such sub-division will not yield ergodic sections if the exposure contains irregularly occurring shocks.

Since many vibration exposures are non-stationary it is clear that neither average nor peak measures provide ideal methods for assessing vibration severity. In the case of severe vibration exposures which may be a hazard to health there are often isolated or repeated shocks. These shocks may sometimes present the greatest risk and it is clearly desirable that their presence is reasonably reflected in the measured values and does not preclude the use of a measurement procedure.

An exceedingly complex time-dependency is defined in International standard $2631(1974,1978)^{1}$ which also advocates the use of root-mean-square time averaging for all vibration measurements. It has been previously indicated that the implicit time-dependency in $\mathrm{rms}$ averaging is very different from that given in the Standard and that some ambiguity therefore arises (Griffin and whitham $(1980)^{2}$ ). This problem was lessened by the inclusion of a crest factor limit of 3.0 since if conditions are appreciably non- 
ergodic the crest factor (peak divided by rms value) will rise. (The limit of 3.0 was raised to 6.0 in ISO 2631 Amendment 1 (1982) $)^{3}$. It follows that the Standard tends to be limited to ergodic motions and has on iy very tentative applicability when the crest factors increase. The Standard cannot be expected to give reasonable values when measuring shocks. Such motions may be among the most likely conditions to present severe discomfort and a health hazard, but Amendment 1 indicates that the Standard may be expected to underestimate their severity.

A simple solution to the problem of assessing non-stationary motions is to employ a cumulative (i.e. dose) measure of vibration severity in preference to an average measure. If appropriate weight can be given to the direction, frequency, magnitude and duration of the motion as it is accumulated there is no need to be concerned with stationarity, ergodicity, or crest factors: the greater the dose the greater the severity of the motion exposure - whatever its pattern or duration.

This paper summarises the definition of what may be the most simple possible vibration dose procedure and illustrates its application to a wide range of vibration environments. The conclusions obtained from the use of the dose procedure are compared with those obtained using procedures based on those in ISO 2631 (1974, 1978) and ISO 2631 Amendment 1 (1982).

The dose procedure employed in this paper commences with the assumption that to maintain the same vibration severity a 16 fold increase in vibration duration should be accompanied by a 2 fold reduction in vibration magnitude. While this original concept was developed from studies of subjective responses to short duration vibration (Griffin and whitham $(1980)^{2}$ ) other considerations suggest that this relation is probably reasonably applicable to repeated shocks and longer duration vibration (Griffin $\left.(1982)^{4},(1984)^{5}\right)$. There are insufficient published data on the health effects of known magnitudes of whole-body vibration to suggest that this method gives a precise or proven time dependency for the prediction of injury or disease. However at very long and at very short durations the time dependency appears more reasonable than that in ISO 2631 (1974, 1978). It is also more simple and avoids the ambiguities of the International Standard. 


\section{DEFINITION OF DOSE}

Vibration Dose: If $a(t)$ is the frequency-weighted acceleration time history (in $\mathrm{ms}^{-2}$ ) at the input to the body then:

$$
\text { Vibration Dose }\left(m^{4} s^{-7}\right)=\int_{t=0}^{t=T} a^{4}(t) d t
$$

The value of the integral may be determined over the full day or that part of the day during which vibration may occur.

If the vibration conditions are constant (or regularly repeated) throughout the day, only one representative period (of duration $t_{1}$ ) need be measured. The total Dose Value for the day will then be the Dose Value for the period $t_{1}$ multiplied by $t_{0} / t_{1}$ (where to is the total period of vibration exposure).

If in a day there are $n$ periods of various durations with measured (or estimated) Dose Values, the total Dose Value for the day is the sum of the individual Dose Values.

The Dose Value may also be calculated from the rmq (root mean quad) of the vibration:

$$
\operatorname{rmq}=\left(\frac{1}{T} \int_{0}^{T} a^{4}(t) d t\right)^{\frac{1}{2}}
$$

The fourth power of the rmq value is multiplied by the duration over which the roq was determined to give the Dose value over this period.

If the crest factor is low and the motion is ergodic it has been suggested that the Dose value of a period of vibration may be estimated from the mo value:

$$
\begin{aligned}
& \text { Estimated } \\
& \text { Dose } \\
& \text { Value } \\
& \text { EDV }\left(\mathrm{m}^{4} \mathrm{~s}^{-7}\right)
\end{aligned}=\left(1.4 \times \mathrm{rms} \text { value, } \mathrm{ms}^{-2}\right)^{4} \times \text { (duration, seconds) }
$$

Relations for specific types of motion have been given elsewhere (Griffin $\left.(1984)^{5}\right)$.

Vibration Dose Values, VDV: Because they depend on the fourth power of the vibration magnitude, Dose Values can become numerically large. The growth in subjective sensation of constant duration stimuli is approximately 
proportional to acceleration magnitude so it is convenient to express the dose by the fourth root of the Dose Value; this is called the Vibration Dose Value (VDV):-

$$
\text { Vibration Dose Value, VDV }\left(\mathrm{ms}^{-1.75}\right)=\left(\int_{t=0}^{t=T} a^{4}(t) d t\right)^{\frac{1}{2}}
$$

The VDV is the rmg magnitude of an equivalent 1 second stimulus. Therefore it is convenient to consider that the Vibration Dose Value gives the magnitude of a 1 second motion which has an equivalent effect. For some motions the Vibration Dose Value may be estimated from the frequency weighted rms value:

Estimated

... Vibration

$$
\begin{aligned}
& \text { Dose } \\
& \text { Value }
\end{aligned} \quad=\left(\left(1.4 \times \mathrm{rms} \text { value, } \mathrm{ms}^{-2}\right)^{4} \times(\text { duration, } \mathrm{s})\right)^{\frac{1}{4}}
$$
EVDV (ms ${ }^{-1.75}$ )

The use of equation (v) allows the presentation of a graphical relation between the rms magnitude and duration of a motion for various Estimated Vibration Dose values. The relation, shown in Figure 1 is exact for Estimated Vibration Dose Values but only approximate for true Vibration Dose Values.

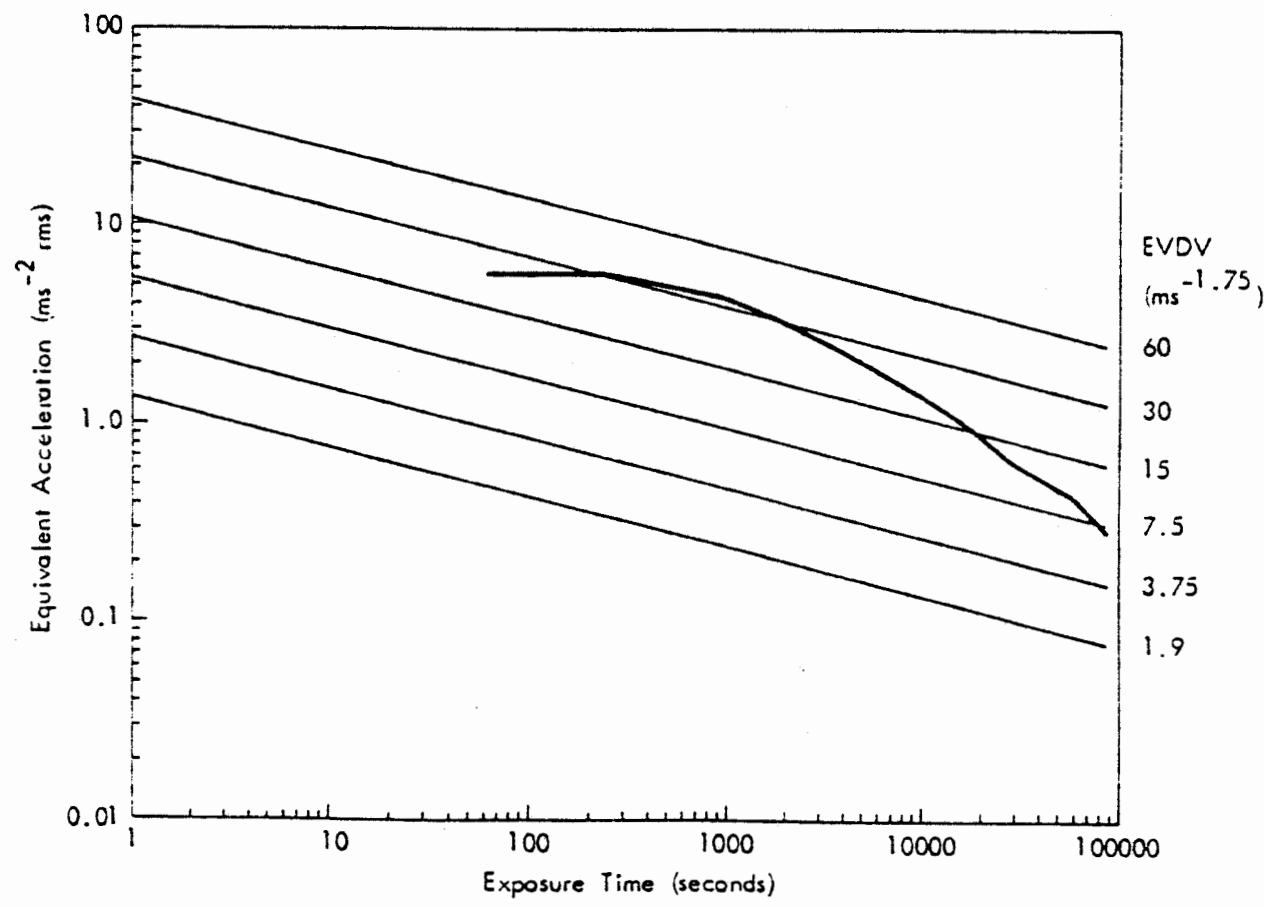

Figure 1: Root mean square acceleration corresponding to Estimated Vibration Dose Values from 1.9 to $60 \mathrm{~ms}^{-1.75}$ compared to the Iso 2631 (1978) exposure limits (comparison limited to 5 to $8 \mathrm{~Hz}$-axis vibration) 
High Vibration Dose Values will cause severe discomfort, pain and injury and indicate, in a general way, the severity of the vibration exposures which caused them. However, with the current Iimitations on the available data on injuries caused by whole-body vibration there can be no consensus of opinion on the precise relation between Vibration Dose values and the risk of injury. It has been suggested that values in excess of about $15 \mathrm{~ms}^{-1.75}$ (a Dose Value of $50000 \mathrm{~m}^{4} \mathrm{~s}^{-7}$ ) will usually cause severe discomfort. It seems reasonable to assume that increased exposure to vibration will be accompanied by increased risk of injury. At high Vibration Dose Values prior consideration of the fitness of the exposed person and the design of adequate safety precautions may be required. The need for regular checks on the health of routinely exposed persons may also be considered.

A comparison of the time dependency with that in ISO 2631 (1978) shows that 15 EVDV ms $\mathrm{ms}^{-1.75}$ gives similar values to the "Exposure Limits" at about $4 \frac{1}{2}$ hours. The rms acceleration corresponding to $15 \mathrm{EVDV} \mathrm{ms} \mathrm{m}^{-2}$ is lower than the ISO 2631 exposure limit between 1 minute and 4h hours; 15 EVD $\mathrm{ms}^{-1.75}$ is at magnitudes above the ISO Exposure Limits at durations in excess of about $4 \frac{1}{2}$ hours.

The above comparison between the two time-dependencies is only valid where the same frequency-weighting is used. There are arguments suggesting that the ISO 2631 z-axis weighting overestimates the importance of vibration from about 1 to $4 \mathrm{~Hz}$ and underestimates the importance of vibration at frequencies in excess of about $12 \mathrm{kz}$. It is therefore currently proposed that Vibration Dose Values should be determined using a new frequency weighting, $W_{b}$, in preference to the ISO 2631 z-axis weighting $w_{g}$. These and other weightings used in this paper are shown in Figure 2 and defined in Table 1. Outside the range 0.5 to $80 \mathrm{~Hz}$ maximal attenuation was provided. (The weighting $W_{b}$ is derived from studies of subjective response to vibration. It is not based on results showing the relative injury potential of different frequencies since such information is not available. It is currently considered that guidance on health effects is most reasonably related to severe discomfort. While other weightings have been derived from other considerations they do not, in general, produce results which are consistent with the average persons expectation of injury when exposed to different vibration frequencies. Weightings based on mechanical impedance, for example, tend to suggest that intolerable 


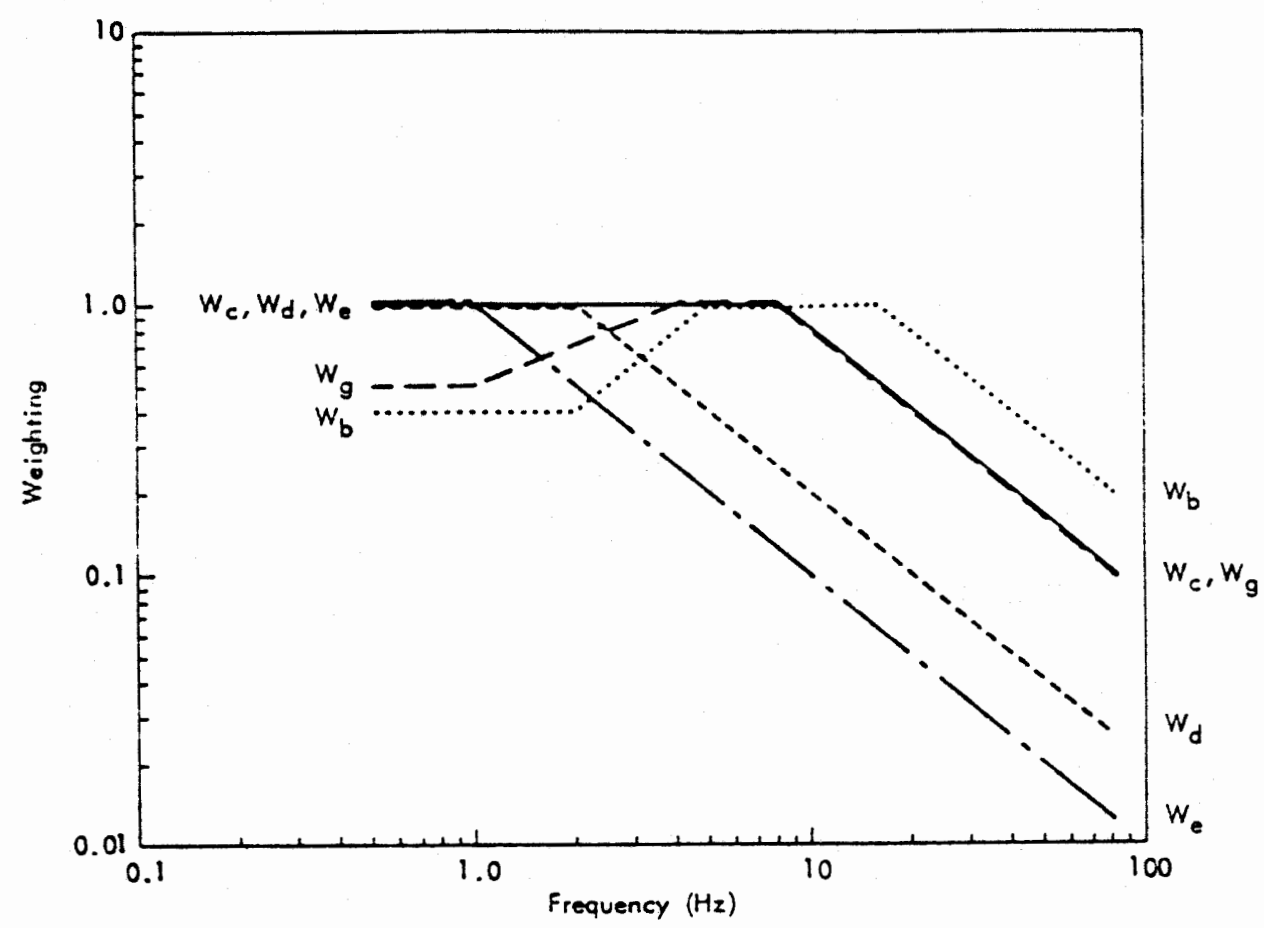

Figure 2: Frequency weightings used in this study

levels of high frequency vibration will cause no adverse effect. The use of the same weighting for considerations of health and comfort is convenient in practice since health and comfort are the prime interests in most assessments.)

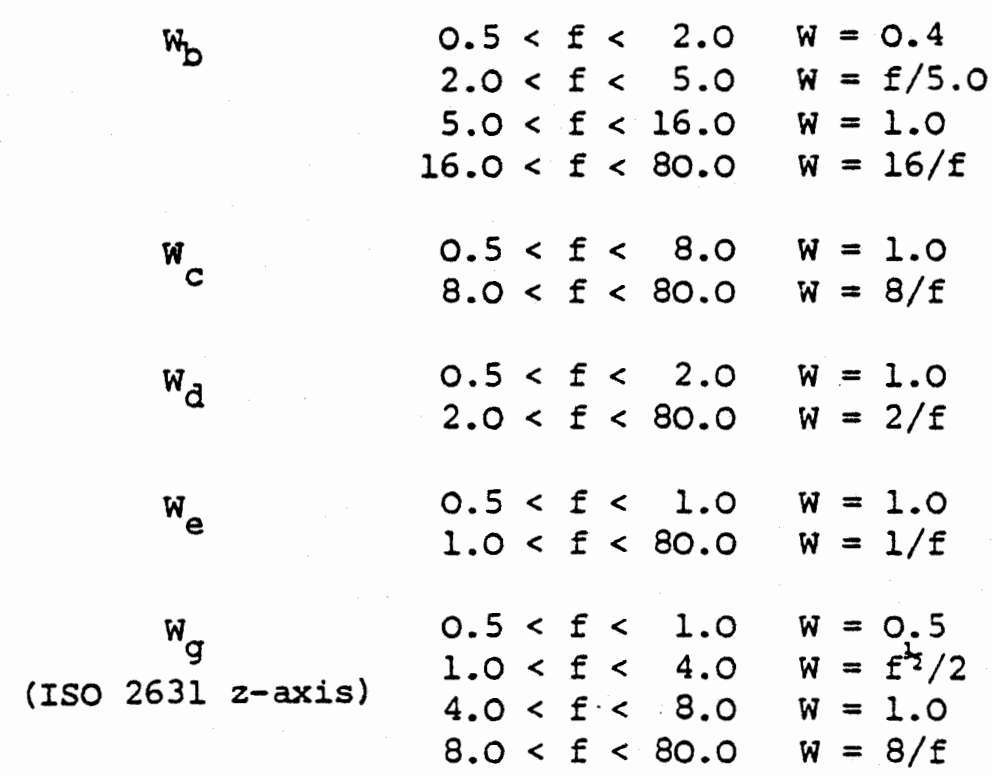

Table 1: Frequency weightings used in this study ( $W=$ weighting; $f=$ frequency, $\mathrm{Hz}$ ) 
The use of the revised weighting for the calculation of Vibration Dose Values alters the comparison of the time dependency with ISO 2631 . For example, at $2 \mathrm{~Hz}$ the revised weighting has a value of 0.4 compared to 0.7071 for the ISO weighting. In consequence, values 768 higher are then acceptable and, for this frequency, an EVDV of $15 \mathrm{~ms}^{-1.75}$ is at a higher rms acceleration than the ISO 2631 Exposure Limits at around 1 minute and for all durations from about $3 / 4$ hour to 24 hours.

\section{EXAMPIES OF VIBRATION DOSE VALUES}

The value of the Vibration Dose (or $\mathrm{rmq}$ ) procedure has been assessed in a number of laboratory studies and generally found to be as good or better than methods based on rms averaging. In this section the method is compared with the use of rms averaging as currently implied in Iso 2631 . The principal purposes of the comparison are to illustrate and assess the method for a range of different environments and consider the extent to which True Vibration Dose Values may be replaced by Estimated Vibration Dose Values determined from measures of the frequency-weighted root-meansquare acceleration.

The examples presented in this paper are based on recordings obtained from various sources (see Acknowledgements). All measurements were obtained at person-seat interfaces or can be reasonably assumed to be a close approximation to vibration at these interfaces. In one case the analysis of the vibration in all 12-axes is presented but for most environments the values shown are restricted to those likely to contribute most to severe discomfort or have an adverse effect on health. All values shown are for vibration frequency weighted according to the weightings and multiplying factors given in the tables. For the revised procedure these weightings and multiplying factors are those which, on current evidence, appear most appropriate in the opinion of the author.

In this paper all values obtained using the weightings from Iso 2631 employ a multiplying factor of 1.4 for seat vibration in the $x$ - and $y$-axes. These values may then be compared directly with those obtained using a multiplying factor of 1.0 for the z-axis. Although the exposure times to exceed the ISO 2631 fatigue-decreased proficiency boundary are shown in the tables they are not discussed: the term 'fatigue-decreased proficiency' is not adequately defined in relation to the complex effects of vibration on performance and there appears to be no reasonable evidence showing that, in general, performance 
depends on the duration of vibration exposure as indicated in ISO 2631. All exposure times based on motions with crest factors in excess of 6.0 are shown in italics.

$=$

A small car: The first example considers the evaluation of the vibration in a small car (see Table 2). The weightings and multiplying factors given for the revised procedure account for the differential sensitivity of the body to vibration frequency in twelve axes ( 3 translational $\left(x_{s}, y_{s}, z_{s}\right.$ ) and 3 rotational $\left(r_{x}, r_{y}, r_{z}\right.$ ) axes on the seat and 3 translational axes at the back $\left(x_{b}, y_{b}, z_{b}\right)$ and feet $\left(x_{f}, y_{f}, z_{f}\right)$. With the moderately low crest factors the approximate discomfort of each axis separately and all axes in combination can be indicated by the rms values and by reference to the semantic comfort scale in Table 3 . This shows that vertical seat vibration, vertical feet vibration and fore-and-aft backrest vibration are the three principal sources of discomfort in this example. In general, weighted values less than about $25 \%$ of the maximum value may be omitted from the calculation of the summed weighted value and this eliminates six axes from this example. The overall discomfort is in the range "a little uncomfortable" to "fairly uncomfortable".

The VDV value concerns severe discomfort and possible effects on health and is restricted to the principal vibration entering the trunk le.g. translational vibration on the seat and fore-and-aft vibration of the backrest). It may be seen that for each axis separately and for the fourth root of the sum of the fourth power of these four VDV values the exposure time before reaching $15 \mathrm{VDV} \mathrm{ms}^{-1.75}$ is greater than 24 hours. It may also be seen that estimated VDV values are in close agreement with true VDV values.

The current IsO procedure assumes that vibration other than in the three translational axes on the seat is insignificant to considerations of comfort or health. The restriction to translational seat vibration differs from the revised procedure which, in accord with common observation, suggests that backrest and footrest vibration may provide a significant contribution to discomfort. For the three axes assessed by the ISO procedure the vertical axis is dominant and, in total, the 'exposure limits' would be exceeded in about 15 hours. The 'reduced comfort boundary' is exceeded in about 45 minutes so, presumably, the ride is to be considered 'comfortable' for shorter periods and 'uncomfortable' for longer periods. 


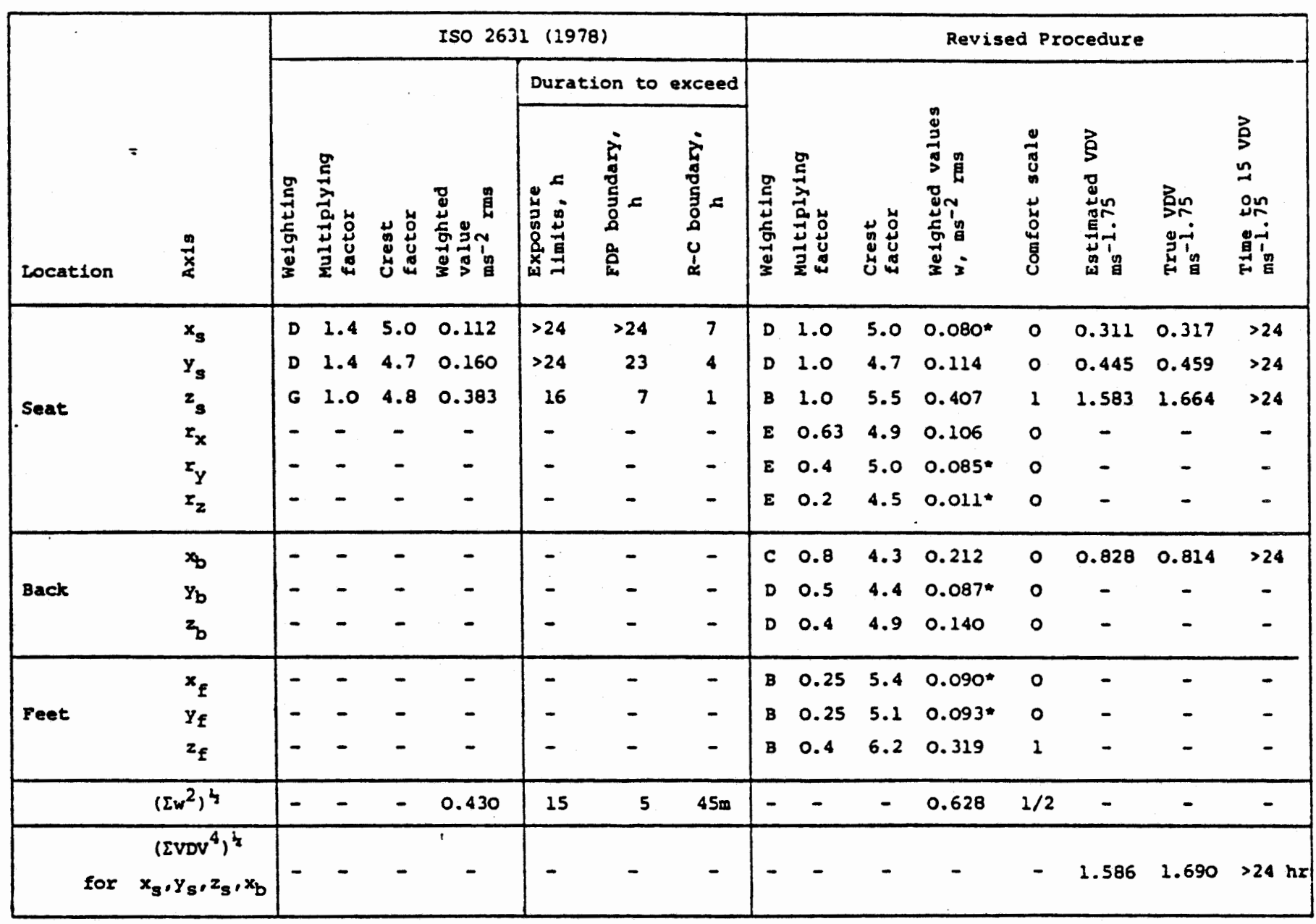

Table 2: Comparison of weighted values from a 60 secording in a small car on a city road using the ISO 2631 and the revised procedure. (* Values less than $25 \%$ of maximum weighted value may be omitted)

Other Road Vehicles: The initial entry in Table 4 is for the measurements in the small car shown in Table 2. The entries according to the revised procedure are restricted to the four axes most likely to contribute to severe discomfort and possibly have an adverse effect on health. The value for the summation over axes (' $\Sigma$ ' in the table) is lowered for root sums of squares because of the omission of the other axes and the comfort rating is altered. The sumation of the VDV over axes is given by the fourth root of the fourth powers of the VDV values in each axis and is therefore dominated by vertical seat vibration and not greatly affected by the restriction to fewer axes of measurement. 


$$
\begin{aligned}
& \text { Weighted } \\
& \text { acceleration } \\
& \mathrm{ms}^{-2} \text { rms } \\
& 0 \text { = not uncomfortable } \\
& 0.2 \\
& \text { L. } 0.315- \\
& 0.4 \\
& 1 \text { = a little uncomfortable } \\
& 2 \text { = fairly uncomfortable }\left[\begin{array}{c}
0.5 \\
0.63
\end{array}\right] \\
& \left.\begin{array}{l}
0.8 \\
1.0
\end{array}\right] 3=\text { uncomfortable } \\
& 4=\text { very uncomfortable }\left[\begin{array}{l}
1.25 \\
1.6
\end{array}\right] \\
& \left.\begin{array}{l}
2.0 \\
2.5
\end{array}\right] 5=\text { extremely uncomfortable }
\end{aligned}
$$

Table 3: Approximate indications of the likely reactions to various magnitudes of weighted vibration.

The van driven over a different test road representative of many ' $A$ ' and ' $B$ ' class roads in Britain exhibited higher values than the small car on the representative city road. Based on an assessment of only four axes the revised procedure indicates a discomfort rating of "fairly uncomfortable". In contrast, the ISO procedure implies that the ride is 'comfortable' for up to 1 minute and 'uncomfortable' for longer periods. Whereas the revised procedure indicates that the ride would not be expected to be hazardous the Iso Exposure Iimits are exceeded in about 5 hours and this therefore represents the ISO "maximum safe exposure".

A different van of similar size was taken on a five hour circular journey (see Table 4 and Griffin $(1980)^{4}$ ). The most notable feature of the values shown are the high crest factors which arise in typical journeys as opposed to the highly constrained test conditions which can be obtained for experimental purposes. The ISO exposure times are not valid at high crest factors and are therefore shown in italics.

The values for the truck driven on a rough test road show, according to the revised procedure, that fore-and-aft seat back vibration was a principal cause of discomfort. This has been confirmed by subjective assessment and laboratory experimentation for this and some other articulated trucks but is ignored in the current ISO procedure. The ISO procedure indicates that 


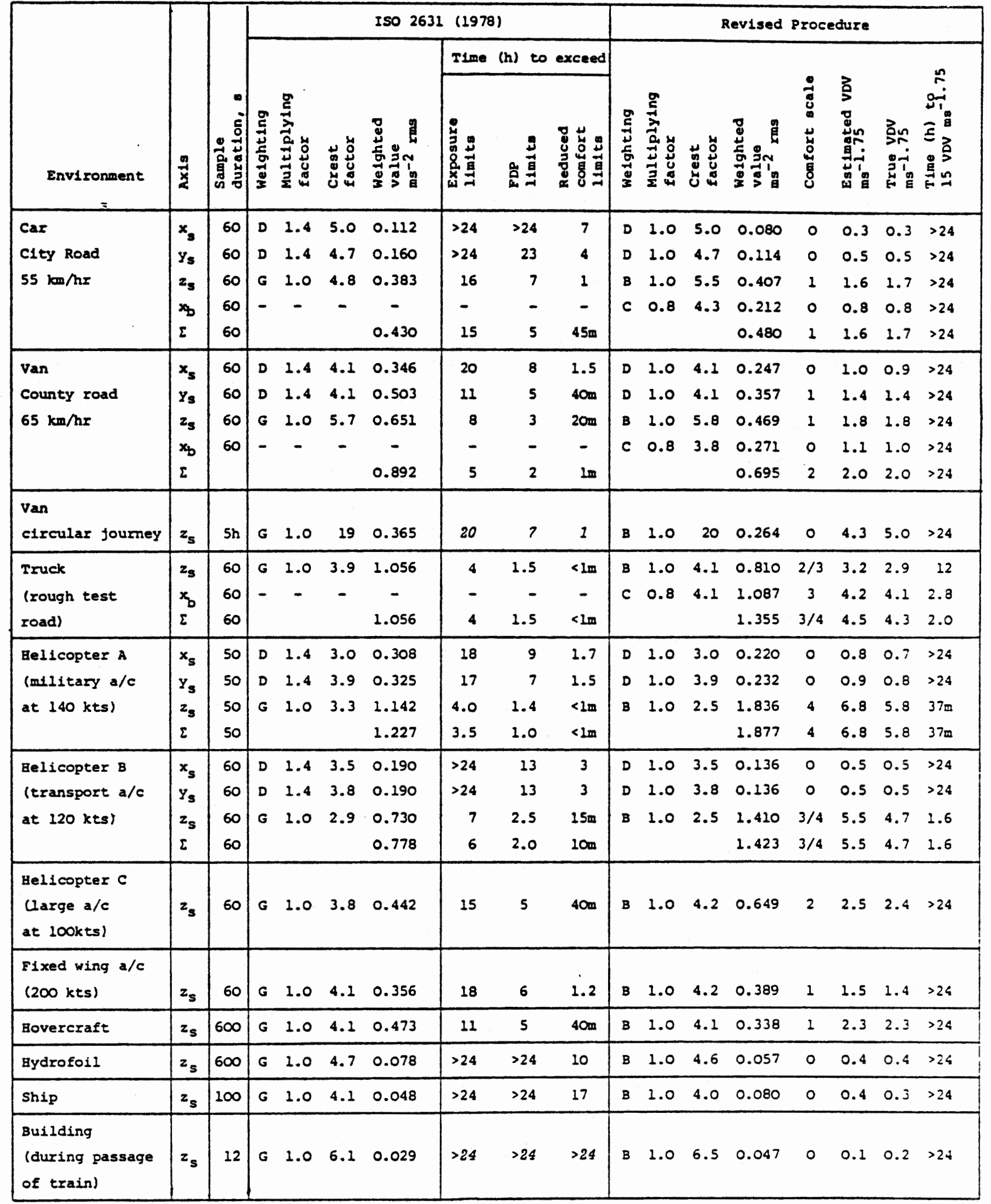

Table 4: Comparison of values obtained using the ISO 2631 and the revised procedure (entries in italics for conditions when crest factor exceeds 6 ). 
the ride is 'uncomfortable' for any duration and that it would be unsafe after about 4 hours. The revised procedure rates the ride as in the range 'uncomfortable' to 'very uncomfortable' and suggests some caution if the exposure time exceeds 2 hours - with this time being dictated by the foreand-aft motion of the backrest not the vertical seat vibration.

Aircraft: of the three helicopters shown in Table 4, helicopters A and B are among those known to produce vibration sufficient to cause interest in pilot and crew well-being. The vibration recordings for these two aircraft were obtained in flight conditions likely to produce above average vibration. For helicopter $A$ the revised procedure suggests the yibration would be judged as 'very uncomfortable' and that some caution should be exercised if this flight condition was repeated for more than about 40 minutes daily. According to the Iso procedure an exposure time of $3 \frac{1}{2}$ hours every day to this condition would be acceptable.

The example of vibration in helicopter B is rated in the range 'uncomfortable' to 'very uncomfortable' and according to the revised procedure would be reasonable for up to about $1 \frac{1}{2}$ hours a day. The Iso procedure suggests the vibration becomes uncomfortable after 10 minutes and that 6 hours exposure to this flight condition per day is acceptable.

Helicopter $\mathrm{C}$ conducts long duration missions and has a lower magnitude of vibration. Both proceduressuggest long durationsof exposure are reasonable. The ISO procedure indicates that vibration becomes uncomfortable after 40 minutes whereas the revised procedure gives the vibration a rating of 'fairly uncomfortable'. The example of fixed wing military aircraft vibration analysed is also low in magnitude. According to the Iso procedure it becomes uncomfortable after 1.2 hours. It is rated as 'a little comfortable' by the revised procedure. Obviously higher values may be obtained in worse weather, at higher speeds or in different aircraft.

Sea Vessels: The hovercraft had a dominant motion at about $0.3 \mathrm{~Hz}$ which is largely removed by the frequency weightings. The motion analysed would be unsafe after 11 hours and uncomfortable after 40 minutes according to the ISo procedure. By the revised procedure this particular motion is not expected to be harmful but would be rated as 'a little uncomfortable'. The motion measured in the hydrofoil was low and judged as 'not uncomfortable' by the revised procedure and as uncomfortable after 10 hours according to Iso 2631. The ship vibration was also of low magnitude only causing discomfort after 17 hours according to ISO 2631 . 
Building Vibration: While vibration in buildings may not normally be expected to cause severe discomfort or injury it would be convenient if similar dose procedures could be applied. Laboratory research reported elsewhere (Woodroof et al $(1983)^{6}$ ) suggest that the dose procedure does appropriately assess subjective reaction to periods of vibration caused by individual trains passing buildings and the method is offered in British standard $6472(1984)^{7}$. The example shown in Table 4 indicates, of course, a relatively low Vibration Dose value for an individual train. The attraction of the method is that it appears to provide the only known method of obtaining a reasonable sumation over a day of vibration which occurs intermittently and for variable periods.

Off-road Vehicles: The vibration of off-road vehicles is often thought to be among the most severe and most likely to cause severe discomfort or injury. Table 5 shows examples obtained from a tractor driven on four surfaces and a military tank driven on a single course at five speeds.

Driving the tractor over the 'smoother track' defined in International Standard 5008 (1979) would be safe for 36 minutes (i.e. about 72 runs) per day according to ISO 2631 but caution would be suggested after 12 minutes (24 runs) per day according to the revised procedure. The track produces discomfort for any period of exposure according to Iso 2631 and 'extreme discomfort' according to the revised procedure.

On the farm road, during hay turning and mowing the limiting Iso exposure times for the tractor are $1.0,1.5$ and 4 hours respectively.compared with 37 minutes, 1 hour and 10 hours for the revised procedure. For these three operations the ISO method judges the ride as uncomfortable for any period. The revised procedure gives ratings ranging from 'extremely uncomfortable' down to 'uncomfortable'.

Vibration measurements on the tractor indicate that lateral seat and foreand-aft backrest vibration are significant. In the tank the examples of vibration shown are for the vertical axis alone. Appreciable increases in vibration severity may be seen as the tank speed is increased. At the lowest speed both the revised procedure and IsO 2631 judge the ride as 'uncomfortable' At the higher speeds the revised procedure shows increased discomfort up to 'extremely uncomfortable'. The Iso procedure has no way of indicating this increased discomfort. 


\begin{tabular}{|c|c|c|c|c|c|c|c|c|c|c|c|c|c|c|c|c|c|}
\hline \multirow[b]{3}{*}{ Environgent } & \multirow[b]{3}{*}{ 离 } & \multirow{3}{*}{ ๑5 } & \multicolumn{7}{|c|}{ ISO 2634 (1978) } & \multicolumn{8}{|c|}{ Rev1sed Procedure } \\
\hline & & & \multirow{2}{*}{\multicolumn{2}{|c|}{ 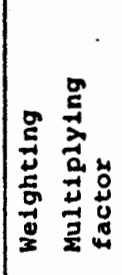 }} & \multirow[b]{2}{*}{ 范 } & \multirow[b]{2}{*}{ 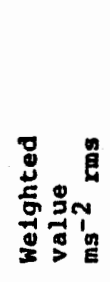 } & \multicolumn{3}{|c|}{ Time $(h)$ to exceed } & \multirow{2}{*}{\multicolumn{2}{|c|}{ 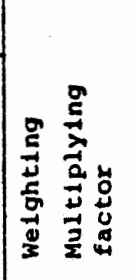 }} & \multirow[b]{2}{*}{ 惑晜 } & \multirow[b]{2}{*}{ 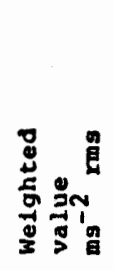 } & \multirow{2}{*}{ 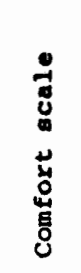 } & \multirow{2}{*}{ 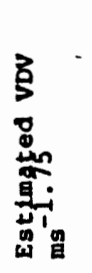 } & \multirow[b]{2}{*}{ 雚品 } & \multirow{2}{*}{ 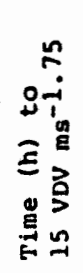 } \\
\hline & & & & & & & 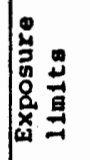 & 品悬 & 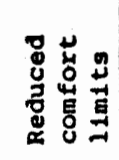 & & & & & & & & \\
\hline \multirow{5}{*}{$\begin{array}{l}\text { Tractor } \\
\text { (ISO } 5008 \\
\text { smoother track } \\
\text { at } 12 \mathrm{~km} / \mathrm{h} \text { ) }\end{array}$} & $x_{3}$ & 25 & D & 1.4 & 3.6 & 0.987 & 5 & 1.5 & $<\operatorname{lm}$ & D & 1.0 & 3.6 & 0.705 & 2 & 2.2 & 2.1 & 16 \\
\hline & $y_{s}$ & 25 & D & 1.4 & 3.5 & 1.862 & 1.6 & 2000 & $<\operatorname{lm}$ & D & 1.0 & 3.5 & 1.330 & $3 / 4$ & 4.2 & 3.9 & 1.5 \\
\hline & $z_{s}$ & 25 & G & 1.0 & 3.0 & 2.112 & 1.3 & $15 m$ & $<1 \mathrm{~m}$ & $\mathbf{B}$ & 1.0 & 3.7 & 1.873 & 4 & 5.8 & 5.7 & $18 m$ \\
\hline & $\infty$ & 25 & - & - & - & - & - & - & - & $c$ & 0.8 & 4.7 & 1.365 & $3 / 4$ & 4.3 & 4.3 & 1.0 \\
\hline & $\Sigma$ & 25 & & & & 2.984 & $36 m$ & $<10$ & $<\ln$ & & & & 2.763 & 5 & 6.5 & 6.4 & $12 \pi$ \\
\hline \multirow{5}{*}{$\begin{array}{l}\text { Tractor } \\
\text { (mowing) }\end{array}$} & $x_{5}$ & 120 & $D$ & 1.4 & 6.7 & 0.587 & 10 & 4 & $25 m$ & $D$ & 1.0 & 6.7 & 0.419 & 1 & 1.9 & 2.1 & $>24$ \\
\hline & $y_{s}$ & 120 & $D$ & 1.4 & 5.2 & 0.802 & 6 & 2 & $10 \mathrm{~m}$ & D & 1.0 & 5.2 & 0.573 & $1 / 2$ & 2.6 & 2.7 & $>24$ \\
\hline & $z_{s}$ & 120 & G & 1.0 & 6.3 & 0.665 & 8 & 3 & $20 m$ & B & 1.0 & 7.6 & 0.609 & $1 / 2$ & 2.8 & 2.9 & 23 \\
\hline & $x_{b}$ & 120 & - & - & - & & - & - & - & $c$ & 0.8 & 5.3 & 0.560 & $1 / 2$ & 2.6 & 2.6 & $>24$ \\
\hline & $\Sigma$ & 120 & & & & 1.195 & 4 & 1 & $<1 m$ & & & & 1.090 & 3 & 3.6 & 3.6 & 10 \\
\hline \multirow{5}{*}{$\begin{array}{l}\text { Tractor } \\
\text { (hay turning) }\end{array}$} & $x_{5}$ & 120 & $D$ & 1.4 & 5.9 & 0.939 & 5 & 1.6 & $<\operatorname{lm}$ & $D$ & 1.0 & 5.9 & 0.671 & 2 & 3.1 & 3.2 & 17 \\
\hline & $y_{s}$ & 120 & $D$ & 1.4 & 3.5 & 1.100 & 4 & 1.4 & $<$ Im & D & 1.0 & 3.5 & 0.786 & 2 & 3.6 & 3.4 & 13 \\
\hline & $z_{s}$ & 120 & G & 1.0 & 8.5 & 1.389 & 2.5 & $40 m$ & $<1 m$ & B & 1.0 & 12.8 & 1.032 & 3 & 4.8 & 5.7 & 1.6 \\
\hline & $x_{b}$ & 120 & - & - & - & - & - & - & - & c & 0.8 & 7.4 & 0.899 & $2 / 3$ & 4.2 & 4.6 & 3.6 \\
\hline & $\Sigma$ & 120 & & & & 2.005 & 1.5 & $20 \mathrm{~m}$ & $<I m$ & & & & 1.715 & 4 & 5.7 & 6.5 & 1.0 \\
\hline \multirow{5}{*}{$\begin{array}{l}\text { Tractor } \\
\text { (farm road) }\end{array}$} & $x_{5}$ & 120 & $D$ & 1.4 & 7.0 & 0.745 & 7 & 2.5 & $35 m$ & $D$ & 1.0 & 7.0 & 0.532 & $1 / 2$ & 2.5 & 2.7 & $>24$ \\
\hline & $y_{s}$ & 120 & $D$ & 1.4 & 4.6 & 1.509 & 2.5 & $40 \mathrm{~m}$ & $<\operatorname{lm}$ & D & 1.0 & 4.6 & 1.078 & 3 & 5.0 & 4.9 & 2.9 \\
\hline & $z_{s}$ & 120 & $G$ & 1.0 & 4.2 & 1.470 & 2.5 & 400 & $<\ln$ & B & 1.0 & 5.5 & 1.292 & $3 / 4$ & 6.0 & 6.4 & 1.0 \\
\hline & $x_{b}$ & 120 & - & - & - & - & - & - & - & C & 0.8 & 6.5 & 0.954 & $2 / 3$ & 4.4 & 4.7 & 3.4 \\
\hline & $\Sigma$ & 120 & & & & 2.235 & 1.0 & $15 m$ & $<1 m$ & & & & 2.006 & $4 / 5$ & 6.9 & 7.2 & $37 m$ \\
\hline Tank $2.4 \mathrm{~ms}^{-1}$ & $z_{s}$ & 30 & $G$ & 1.0 & 3.3 & 1.299 & 3 & $40 \mathrm{~m}$ & $<\operatorname{lm}$ & $B$ & 1.0 & 3.6 & 1.027 & 3 & 3.4 & 3.3 & 3.5 \\
\hline rough $3.11 \mathrm{~ms}^{-1}$ & $z_{5}$ & 30 & G & 1.0 & 3.6 & 1.466 & 2.5 & 35m & $<\operatorname{lm}$ & $B$ & 1.0 & 3.7 & 1.143 & 3 & 3.7 & 3.7 & 2.3 \\
\hline $\begin{array}{ll}\text { test } & 3.53 \mathrm{~ms}^{-1} \\
\text { course } & \end{array}$ & $z_{s}$ & 30 & G & 1.0 & 3.6 & 1.744 & 2 & $25 m$ & $<\ln$ & B & 1.0 & 3.9 & 1.336 & $3 / 4$ & 4.4 & 4.3 & 2.25 \\
\hline $3.87 \mathrm{~ms}^{-1}$ & $z_{s}$ & 30 & G & 1.0 & 4.7 & 1.948 & 1.5 & $20 \mathrm{~m}$ & $<1 \mathrm{~lm}$ & B & 1.0 & 4.5 & 1.495 & $3 / 4$ & 4.9 & 4.8 & $46 m$ \\
\hline $4.1 \mathrm{~ms}^{-1}$ & $z_{s}$ & 30 & $G$ & 1.0 & 11.5 & 2.251 & 1.2 & $15 m$ & $<1 m$ & B & 1.0 & 16.1 & 1.764 & 4 & 5.8 & 9.9 & $2.4 \mathrm{~m}$ \\
\hline $4.46 \mathrm{~ms}^{-1}$ & $z_{s}$ & 30 & G & 1.0 & 14.4 & 2.504 & 1 & $8 m$ & $<1 m$ & B & 1.0 & 21.2 & 2.015 & $4 / 5$ & 6.6 & 14.5 & $32 \mathrm{~s}$ \\
\hline
\end{tabular}

Table 5: Comparison of values obtained using the ISO 2631 and the revised grocedure (entries in italics for conditions when crest factor exceeds 6 ). 
At the lower tank speed both procedures indicate that about 3 hours would be a reasonable daily exposure time before becoming concerned about the health of the tank crew. As the speed and vibration increase the revised procedure suggests a somewhat more rapid curtailment of the daily exposure time - down to 46 minutes at $3.87 \mathrm{~ms}^{-1}$. At higher speeds the crest factor exceeds 6.0 and the ISO exposure times are not valid. They indicate, for example, an allowable exposure time of 1 hour at $4.46 \mathrm{~ms}^{-1}$ while the revised procedure suggests that caution should be exercised if more than about one 30 second run is undertaken per day. (It is understood that the latter is more in accord with the views of the tank crew).

\section{DISCUSSION}

Although 22 recordings (and 53 channels of data) have been analysed it cannot be assumed that all measurements are typical of the vibration environments in which they have been obtained. vibration conditions vary greatly with speed, ground condition, flight condition etc. and it is not meaningful to label a system as having particular characteristics without more complete specifications of the relevant variables. However the measurements presented have not been selected using any criteria which would make them unrepresentative and it appears reasonable to use them to assess the characteristics of the Vibration Dose Value and ISO 2631 methods of assessing vibration severity.

The two procedures differ in several respects: time dependency, crest factor limitation, frequency weighting, number of axes, the method of summation over axes, the rating of the degree of discomfort and the meaning of 'exposure Iimits'.

The ISO time dependency has a very rapid decline in acceptable magnitude at durations in excess of an hour or so. In consequence very low limits are specified for long durations and only the very low magnitudes in the hydrofoil, ship and building fell below the limits which the Iso standard defines as the maximum safe vibration for a 24 hour period. In contrast, at short durations, the standard allows high magnitudes and the shortest allowable exposure time determined in this study was 36 minutes. For durations below about 4 minutes the absence of any time dependency appears unreasonable and is somewhat in conflict with the use of root mean square measurement of vibration magnitude. In general, it not clear when root mean square averaging should be used and when the ISO time-dependency should be employed. 
The ISO 2631 time dependency presents other problems at long durations. For example Draft Addendum 1 (1980) to ISO 2631 indicates that with continuous vibration in workshops at half the 24 hour exposure limit there would only be major complaints if no prior warning was not given. Vibration limits for ships in a proposed Draft Addendum 3 to ISO 2631 which have been based on crew subjective assessments are in excess of the ISO 263124 hour exposure limits! In addition, the very complex form of the time dependency and the numerical errors in the Tables of both the 1974 and 1978 versions of the Standard tend to frustrate its use.

The Vibration Dose Value of $15 \mathrm{~ms}^{-1.75}$ has been suggested as an approximate 'action level'. This value could have some impact on the operation of the truck, helicopter, tractor and tank. For these and the other environments the exposure durations before $15 \mathrm{~ms}^{-1.75}$ is exceeded appear broadly consistent with the conditions which occurred during the measurement periods. With the truck and the tractor longer times would have been found if backrest vibration had not been assessed.

The ISO 2631 method was originally limited to a crest factor of 3 and it is clear that this was set arbitrarily without knowledge of the crest factorswhich normally occur - only two of the 45 measurements obtained with the ISO weightings had a crest factor below 3! The raising of the crest factor to 6 in ISO AMI (1982) brought the majority of the current measurements within the scope of the standard. However the important tractor and tank operations tend to exceed this limit. In addition, when measurements are made for lengthy periods under true operational conditions (e.g. the 5 hour recording in the van) very much higher crest factors tend to occur.

The Vibration Dose Value procedure only discriminates between low and high crest factors in suggesting that Estimated Vibration Dose Values (calculated from rms measurements) may be used when the crest factor is below about 6 . The values shown in the Tables indicate that in general the Estimated Vibration Dose Values gave remarkably accurate predictions of the true values. Apart from the few high crest factor environments the only notable deviation occurs with two of the helicopter where the nearsinusoidal motion results in a slight over-estimate of the Vibration Dose value. It therefore appears that for most environments the Vibration Dose value procedure may be used with rms measures of vibration magnitude. This may slightly overestimate vibration severity in a few exceptional very low crest factor environments and will only significantly underestimate the 
severity when the crest factor is very high.

The effect of the different frequency weightings used for z-axis vibration may be assessed by comparing the weighted rms values given by the two procedures. The $W_{b}$ weighting used in the revised procedure tends to reduce the importance of vibration below $5 \mathrm{~Hz}$ but increase the importance of vibration above $8 \mathrm{~Hz}$. In consequence the weighted values in the truck, tractor and tank are somewhat reduced while those in the helicopters are increased by the revised procedure. For helicopters $A$ and $B$ the exposure duration required to achieve a Vibration Dose value of $15 \mathrm{~ms}^{-1.75}$ was also calculated using the ISO 2631 weighting. This increased the times from 37 minutes to 3.3 hours for helicopter $A$ and from 1.6 hours to 21.8 hours for helicopter B. For these two helicopters, which are known to be among the worst in service, the exposure times calculated with the Iso 2631 weighting do not seem reasonable.

The use of weighting $W_{b}$ for z-axis seat vibration also makes the vibration Dose value procedure compatible with the generalised procedure for assessing comfort with up to 12 axes of vibration. While a 12 axis procedure may superficially appear complex it employs only four different weightings and will generally be simplified to only those axes in which there is significant motion. The results presented here illustrate that measurements of the three translational axes of vibration on the seat squab may not give good overall indications of vibration discomfort. It seems reasonable to restrict the Vibration Dose Value procedure to these three axes and fore-and-aft seat vibration since these are the dominant inputs to the torso. Where there is no seat back (or no contact with the seat back) measurements in this axis would not be appropriate.

Different methods of summation over axes are defined by the two procedures. Originally Iso 2631 specified the consideration of only the most severe axis but this was changed by Amendment I (1982) to the use of the root sum of squares of the three axes of seat vibration. Tables 3 and 4 show that in some cases this appreciably reduces the allowable exposure times. The Vibration Dose Value procedure employs the summation of doses (i.e. the fourth root of the sums of the fourth powers of the VDV's) and is therefore more likely to be dominated by the measurements in the most severe axis.

It has been seen that the scale of discomfort suggested in the revised procedure offers a means of grading the severity of the likely sensations 
produced by the vibration. In contrast, the current ISo 'reduced comfort boundary' offers an indication of the duration after which the vibration is supposed to become uncomfortable - and in many cases it can only be concluded that it is some time less than 1 minute. The indications offered by the Vibration Dose Value procedure relate to severe discomfort and possible injury: they suggest caution but do not imply a limit. The ISO 'exposure limit' defines the "maximum safe exposure" and allows little flexibility.

\section{CONCLUSIONS}

Examples of the use of a proposed revised procedure for assessing whole-body vibration have shown that without exception it produces very reasonable indications of vibration severity. It has been shown that in most cases the procedure may be used with root mean square measures of vibration magnitude. However the same basic procedure may be calculated differently to assess high crest factor, repeated shock and intermittent vibration.

It appears that approximately $15 \mathrm{~ms}^{-1.75}$ is an appropriate vibration Dose Value at which some consideration of the effects on the health of routinely exposed persons may be appropriate. By reference to equation ( $v$ ) it may be seen that for vibration which does not have a high crest factor the exposure time (in hours) before this is exceeded is given by:

$$
\text { Exposure time to } 15 \mathrm{~ms}^{-1.75} \approx \frac{3.7}{\mathrm{a}^{4}} \text { hours }
$$

where the $\mathrm{a}$ is the rms magnitude $\left(\mathrm{ms}^{-2}\right)$ of the vibration which has been appropriately frequency-weighted.

With ever-increasing understanding of the response of the body to vibration there will, no doubt, be further suggestions on how the proposed revised procedure may be improved. Changes of detail could alter the appearance of the method but the basic concept seems correct and useful. It now appears most appropriate that it should be used more widely so as to further assess both the principal and the detail.

\section{ACKNOWLEDGEMENTS}

The author would like to acknowledge the assistance provided by the National Institute of Agricultural Engineering, the Royal Army Research and Development 
Establishment, the Royal Aircraft Establishment and colleagues at the Institute of Sound snd Vibration Research.

\section{REFERENCES}

1. International Organisation for Standardisation

'Guide for the evaluation of human exposure to whole-body vibration', ISO 2631, 1974, 1978.

2. Griffin, M.J. and Whitham, E.M.

'Time dependency of whole-body vibration discomfort', JASA $68(5), 1522-1523,1980$.

3. Intemational organisation for standardisation

'Guide for the evaluation of human exposure to whole-body vibration', Amendment 1, ISO 2631 - 1978/A1 - 1982.

4. Griffin, M.J.

The definition of hazard dose values for whole-body vibration and shock', U.K. Informal Group Meeting on Human Response to Vibration held at Health and Safety Executive, Cricklewood, London, 16-17 September 1982.

5. Griffin, M.J.

'A single number dose procedure for whole-body vibration and repeated shock', Permanent Commission and International Association on Occupational Health - meeting at USSR Academy of Medical Sciences, 12-16 March 1984.

6. Woodroof, H.J., Lewis, C.H. and Griffin, M.J. 'Experimental studies of subject response to railway-induced building vibration', ORE Utrecht, Technical Document DT159, September 1983.

7. British Standards Institution

'The evaluation of human exposure to vibration in buildings ( $1 \mathrm{~Hz}$ to $80 \mathrm{~Hz}$ )', BS 6472,1984 . 